Further studies are needed on utilisation and linkage to care and prevention services.

\section{P17.03 CONVENIENT HIV TESTING SERVICE MODELS COMBINED WITH RAPID TESTING ARE ATTRACTING PREVIOUSLY UNTESTED GAY AND BISEXUAL MEN}

${ }^{1,2} \mathrm{~V}$ Knight*, ${ }^{2} \mathrm{H}$ Wand, ${ }^{3} \mathrm{~J}$ Gray, ${ }^{2} \mathrm{P}$ Keen, ${ }^{1,4} \mathrm{~A}$ McNulty, ${ }^{2} \mathrm{R}$ Guy. ${ }^{1}$ Sydney Sexual Health Centre, South East Sydney Local Health District, Sydney, 2000, Australia; ${ }^{2}$ The Kirby Institute, University of New South Wales, Sydney, NSW, 2052, Australia; ${ }^{3}$ ACON Health, 414 Elizabeth Street Surry Hills, Sydney, NSW, 2010, Australia; ${ }^{4}$ School of Public Health and Community Medicine, University of NSW, Kensington, NSW, 2052, Australia

\section{$10.1136 /$ sextrans-2015-052270.581}

Introduction HIV testing is a cornerstone of the treatment as prevention (TasP) approach. In Australia, gay and bisexual men (GBM) account for the majority of HIV diagnoses each year, but less than a quarter are testing at the recommended frequency. In response, a range of new service delivery models were introduced in Sydney over the past few years. We assessed which HIV testing service delivery models were more likely to attract GBM who had never previously tested or were testing infrequently.

Methods We compared demographics, risk behaviour and HIV testing history among new GBM clients attending three different HIV testing service models (fast-track Xpress clinic, fixed-site community-based service and time-limited community-based shopfront) between August 2013 and May 2014. All services offered HIV rapid testing. We used multivariate regression to assess factors (including service model) associated with being untested or infrequent testers (not tested within the past 12 months).

Results Overall, 1704 new GBM attended the services; 19\% were untested and $41 \%$ were infrequent testers. Across the services, there were significant differences in demographics, risk behaviour and past HIV testing history. The overall HIV seropositivity was $1.2 \%(95 \% \mathrm{CI}: 0.8 \%-1.9 \%)$ and STI positivity was $12.4 \%$ (95\% CI: 11.6-17.2) with no significant differences across services. Factors independently associated with being untested were attendance at the two community sites, younger age, being born in Asia, living in North Sydney, being bisexual and reporting fewer male sexual partners. Factors independently associated with infrequent testers were attending the fast track Xpress clinic, being older, being born in Asia and reporting fewer male partners.

Conclusion The findings show the two community sites reached more untested men and the fast-track clinic model more infrequent testers but the HIV/STI diagnosis rate was consistent across services, indicating that all three testing models are important to increase HIV testing among GBM.

Disclosure of interest statement Vickie Knight is supported by an Australian Postgraduate Association scholarship. No pharmaceutical grants were received in the development of this study.
P17.04 THE RELATIONSHIP BETWEEN HIV TESTING FREQUENCY AND HIV RISK PERCEPTION AMONG PERUVIAN MSM AND TRANSGENDER WOMEN

${ }^{1}$ Sk Vargas*, ${ }^{1,2}$ KA Konda, ${ }^{1}$ SR Leon, ${ }^{3} \mathrm{G}$ Calvo, ${ }^{4} \mathrm{HJ}$ Salvatierra, ${ }^{5} \mathrm{~B}$ Brown, ${ }^{2} \mathrm{JD}$ Klausner, ${ }^{1}$ CF Caceres. 'Unit of Health, Sexuality and Human Development, and Laboratory of Sexual Health, Universidad Peruana Cayetano Heredia, Lima, Peru; ${ }^{2}$ University of California, Los Angeles, CA, USA; ${ }^{3}$ Epicentro, Lima, Peru; ${ }^{4}$ Alberto Barton Health Center, Health Directionate of Callao, Lima, Peru; ${ }^{5}$ University of California, Invine, CA, USA

\subsection{6/sextrans-2015-052270.582}

Introduction HIV in Peru is concentrated among men who have sex with men (MSM) and transgender women (TW). Over 70\% of HIV+ MSM/TW ignore their status, as HIV testing frequency is low, delaying diagnosis and treatment. We aimed to assess the relationship between HIV testing frequency and perceived HIV risk among MSM/TW.

Methods This analysis included baseline data from MSM/TW who reported a negative or unknown HIV status from a STI clinic cohort of MSM/TW at high risk in Lima. The behavioural survey assessed HIV risk perception (high, moderate, low) and prior testing frequency (testing was defined as frequent if it had occurred at least biannually). For HIV diagnosis, we used a $3^{\text {rd }}$ generation rapid test, a $4^{\text {th }}$ generation EIA, and Western Blot confirmation. Chi-square tests and multivariable regression were used to estimate adjusted prevalence ratios (aPRs).

Results Among eligible subjects (243 MSM and $67 \mathrm{TW}), 122$ (39\%) reported frequent prior HIV testing, while HIV risk was perceived as high, moderate and low by 72 (23\%), 148 (48\%) and, 90 (29\%) participants, respectively. Frequent HIV testing was more prevalent among participants reporting low/no HIV risk (aPR: 1.62; CI: 1.20-2.20) and among those reporting previous syphilis infection (aPR: 1.58; CI: 1.22-2.04); but was less prevalent among participants reporting recent condomless anal intercourse (aPR: 0.67; CI: 0.51-0.90). Baseline HIV status and HIV risk perception were not associated: HIV prevalence was $11 \%, 13 \%$ and $10 \%$ among those reporting high, moderate and low/no HIV risk (chi-square p-value $>0.1$ ).

Conclusion Frequent HIV testing was more prevalent among MSM/TW reporting low/no HIV risk. Risk perception did not correlate with HIV status among previously undiagnosed subjects. WHO-recommended biannual HIV testing should be promoted, and factors potentially affecting risk perception and testing frequency (e.g. access, stigma) should be identified and addressed by HIV programming for MSM/TW in Peru.

\section{P17.05 HIV TEST REFUSALS AMONG BLACK AFRICANS ATTENDING SEXUALLY TRANSMITTED INFECTION CLINICS IN ENGLAND, 2013}

H Mohammed*, M Furegato, A Nardone, G Hughes. HIV \& STI Department, Public Health UK

\subsection{6/sextrans-2015-052270.583}

Introduction Black Africans comprise the second largest group affected by HIV in England, but it is estimated that 34\% are 